\title{
Neuronal Intranuclear Inclusion Disease Presenting with Resting Tremor
}

\author{
Naoyuki Kitagawa ${ }^{a} J_{\text {Jn Sone }}^{c}$ Gen Sobue $^{c}$ Masahiko Kuroda $^{b}$ \\ Michio Sakurai ${ }^{\mathrm{a}}$ \\ ${ }^{a}$ Department of Neurology, Kohsei Chuo General Hospital, and ${ }^{b}$ Department of Molecular \\ Pathology, Tokyo Medical University, Tokyo, and 'Department of Neurology, Nagoya \\ University Graduate School of Medicine, Nagoya, Japan
}

\section{Key Words}

Neuronal intranuclear inclusion disease $\cdot$ Tremor · Skin biopsy

\begin{abstract}
Neuronal intranuclear inclusion disease (NIID) is a rare neurodegenerative disease with various neurological symptoms. A 73-year-old woman presented with slowly progressive tremor in both hands. The resting tremor was enhanced by cognitive activity and walking. However, there were no other signs of parkinsonism. Levodopa and trihexyphenidyl were ineffective against the tremor. A diagnosis of NIID was made based on skin biopsy findings. The tremor in this case was very similar to that seen in Parkinson's disease (PD). Previous reports and the present case indicate that NIID patients can develop tremor that is similar in character to that seen in PD. NIID should be considered in the differential diagnosis of resting tremor similar to PD.

(c) 2014 S. Karger AG, Basel
\end{abstract}

\section{Background}

Neuronal intranuclear inclusion disease (NIID) is a rare neurodegenerative disease characterized pathologically by the presence of eosinophilic hyaline intranuclear inclusions in neuronal and somatic cells $[1,2]$. A diagnosis of NIID can be made by skin biopsy in the antemortem period [3]. Previously reported cases of NIID showed various neurological symptoms, including pyramidal and extrapyramidal symptoms, cerebellar ataxia, dementia, convulsion, neuropathy, and autonomic dysfunction [1-4]. A few cases of NIID with 
Kitagawa et al.: Neuronal Intranuclear Inclusion Disease Presenting with Resting Tremor

parkinsonism have also been reported $[2,5,6]$. Here, we report a case of NIID showing resting tremor without other extrapyramidal signs.

\section{Case Presentation}

A 73-year-old Japanese woman with a history of hypertension and hyperlipidemia was referred to our hospital because of bilateral hand tremor. The tremor appeared bilaterally from the onset. She had no family history of neurological disease. A diagnosis of essential tremor was made, but arotinolol hydrochloride at a dose of $20 \mathrm{mg}$ /day was ineffective. After 2 years, she was again referred to our hospital due to staggering and aggravation of hand tremor. Neurological examination showed resting, positional, and intentional tremor in both upper limbs. The resting tremor was enhanced by cognitive activity and walking (online suppl. video, www.karger.com/doi/10.1159/000363687). She showed neither bradykinesia nor rigidity, and her gait was slightly unsteady but not ataxic. No other neurological abnormalities were found. Surface electromyography of the right common digital extensor muscle and ulnar flexor muscle showed discharges of muscle activity at frequencies of 6 and $3 \mathrm{~Hz}$, respectively. Brain magnetic resonance imaging (MRI) revealed dilation of the lateral ventricle, moderate cerebral atrophy, and high-intensity areas in the cerebral white matter in T2-weighted and fluid-attenuated inversion recovery images. MRI diffusion-weighted imaging (DWI) showed a high-intensity signal in the corticomedullary junction (fig. 1). ${ }^{99 \mathrm{mTc}}$ Tc L,L-ethyl cysteinate dimer (ECD) single-photon computed emission tomography (SPECT) showed normal uptake in the thalamus and basal ganglia (fig. 2). Cerebrospinal fluid was normal, and blood examination showed normal thyroid function. Cardiac uptake of 123]metaiodobenzylguanidine (MIBG) during myocardial scintigraphy was normal (H/M ratio early: 2.55, delayed: 2.49). We performed skin biopsy as part of the differential diagnosis between leukoencephalopathy and NIID. Immunofluorescence analysis with anti-ubiquitin antibody revealed intranuclear inclusions in the adipocytes, sweat gland cells, and fibroblasts (fig. 3). A diagnosis of NIID was made based on the skin biopsy findings [3]. The patient was treated with levodopa at $300 \mathrm{mg} /$ day and trihexyphenidyl at $6 \mathrm{mg}$ per day, which did not improve tremor.

\section{Discussion}

Our patient showed resting, positional, and intentional tremor, similar to the tremor seen in Parkinson's disease (PD), in the bilateral upper limbs. The resting tremor in PD patients typically has a frequency between 4 and $6 \mathrm{~Hz}$ and is enhanced by motor or cognitive activity $[7,8]$. However, extrapyramidal signs, including bradykinesia or rigidity, were not seen in this case. About half of all PD patients show tremor as the initial symptom [9]. In some patients, tremor remains relatively unchanged, and non-tremor components of parkinsonism are mild [10]. However, the tremor in our case was resistant to medication, and uptake of 123I-MIBG during myocardial scintigraphy, which is known to be decreased in PD, was normal [11]. It is therefore unlikely that the resting tremor in this case was due to PD. The pathophysiology of PD has not been fully elucidated, but it has been hypothesized that the pacemaker of resting tremor exists in the basal ganglia-thalamocortical circuits [7, 8]. A few cases of NIID with parkinsonism have been reported [2, 5, 6]. O'Sullivan et al. [5] reported juvenile parkinsonism of NIID. Their case showed asymmetric arm tremor and bulbar symptoms and responded well to levodopa. Widespread hyaline intranuclear 
inclusions and neuronal depletion in the substantia nigra were observed at autopsy [5]. Munoz-Garcia et al. [6] reported an autopsy case that developed tremor and unsteady gait as the first symptoms at the age of 50 years. This case also showed writhing movements of the head, trunk, and limbs, as well as repetitive outbursts of anger. Postmortem examination revealed severe neuronal loss in the caudate and putamen but not in the substantia nigra or globus pallidus. The thalamus, mesencephalon, pons, and medulla were histologically normal, except for nuclear inclusions in glial cells. Inclusions were also found in the dentate nuclei of the cerebellum. The authors suggested that functionally injured neurons in the dentate nuclei may be related to the tremor [6]. In contrast, in our case, the onset was later in life. The tremor was resistant to medication, and no other parkinsonian symptoms were seen. Sone et al. [12] reported that high signal intensity in the corticomedullary junction in DWI was a highly characteristic finding in NIID, and skin biopsy was useful for diagnosis. We also made a diagnosis of NIID based on the findings of MRI and skin biopsy. These previous reports and the present case suggest that NIID patients can develop tremor that is similar to that seen in PD. Therefore, NIID should be considered in the differential diagnosis of resting tremor.

\section{References}

1 Sone J, Hishikawa N, Koike H, et al: Neuronal intranuclear hyaline inclusion disease showing motor-sensory and autonomic neuropathy. Neurology 2005;65:1538-1543.

2 Takahashi-Fujigasaki J: Neuronal intranuclear hyaline inclusion disease. Neuropathology 2003;23:351-359.

- 3 Sone J, Tanaka F, Koike H, Inukai A, Katsuno M, Yoshida M, Watanabe H, Sobue G: Skin biopsy is useful for the antemortem diagnosis of neuronal intranuclear inclusion disease. Neurology 2011;76:1372-1376.

4 Zannolli R, Gilman S, Rossi S, et al: Hereditary neuronal intranuclear inclusion disease with autonomic failure and cerebellar degeneration. Arch Neurol 2002;59:1319-1326.

-5 O'Sullivan JD, Hanagasi HA, Daniel SE, Tidswell P, Davies SW, Lees AJ: Neuronal intranuclear inclusion disease and juvenile parkinsonism. Mov Disord 2000;15:990-995.

6 Munoz-Garcia D, Ludwin SK: Adult-onset neuronal intranuclear hyaline inclusion disease. Neurology 1986;36:785-790.

7 Helmich RC, Toni I, Deuschl G, Bloem BR: The pathophysiology of essential tremor and Parkinson's tremor. Curr Neurol Neurosci Rep 2013;13:378.

8 Dovzhenok A, Rubchinsky LL: On the origin of tremor in Parkinson's disease. PLoS One 2012;7:e41598.

-9 Martin WE, Loewenson RB, Resch JA, Baker AB: Parkinson's disease: clinical analysis of 100 patients. Neurology 1973;23:783-790.

10 Josephs KA, Matsumoto JY, Ahlskog JE: Benign tremulous parkinsonism. Arch Neurol 2006;63:354-357.

11 Mitsui J, Saito Y, Momose T, Shimizu J, Arai N, Shibahara J, Ugawa Y, Kanazawa I, Tsuji S, Murayama S: Pathology of the sympathetic nervous system corresponding to the decreased cardiac uptake in ${ }^{123}$ [metaiodobenzylguanidine (MIBG) scintigraphy in a patient with Parkinson disease. J Neurol Sci 2006;243:101-104.

12 Sone J, Kitagawa N, Sugawara E, Iguchi M, Nakamura R, Koike H, Iwasaki Y, Yoshida M, Takahashi T, Chiba S, Katsuno M, Tanaka F, Sobue G: Neuronal intranuclear inclusion disease cases with leukoencephalopathy diagnosed via skin biopsy. J Neurol Neurosurg Psychiatry 2014;85:354-356. 


\section{Case Reports in Neurology}
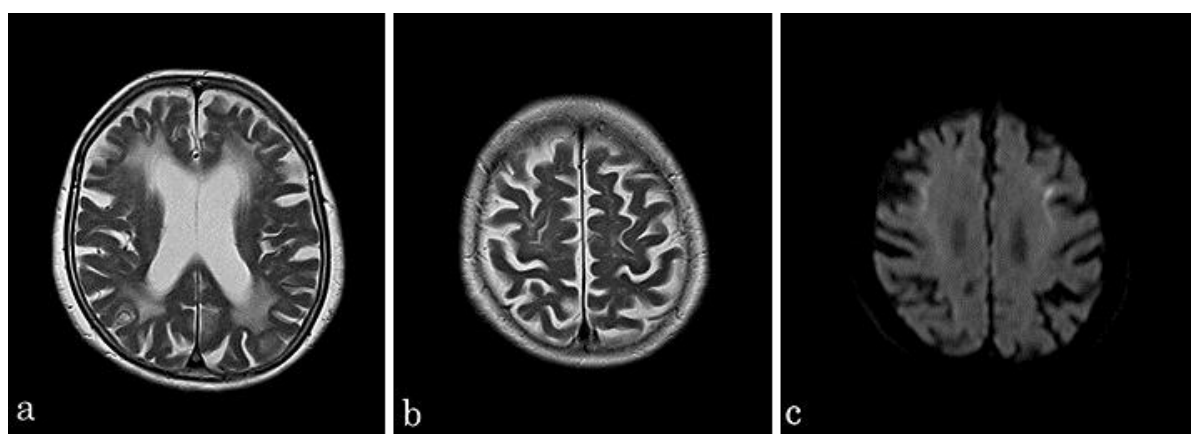

Fig. 1. a, b T2-weighted MRI showing dilation of the lateral ventricle, moderate cerebral atrophy, and high-intensity areas in the cerebral white matter. c DWI indicated high signal intensity in the corticomedullary junction.

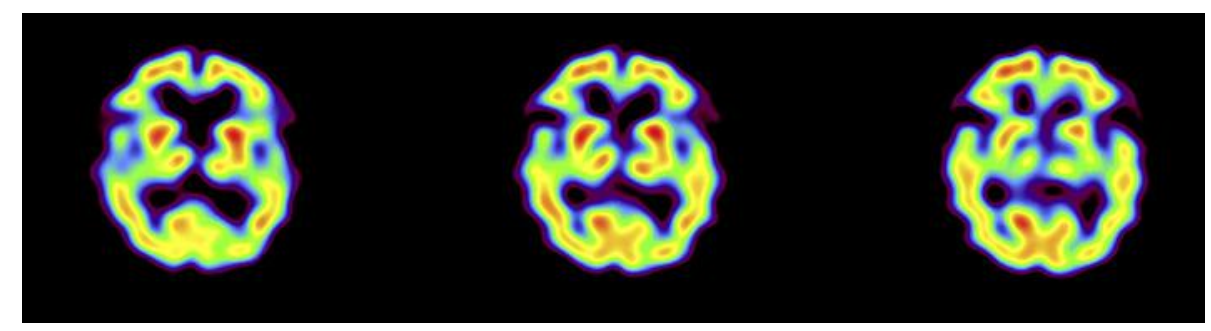

Fig. 2. Normal uptake of the basal ganglia in ECD SPECT. 


\section{Case Reports in Neurology}
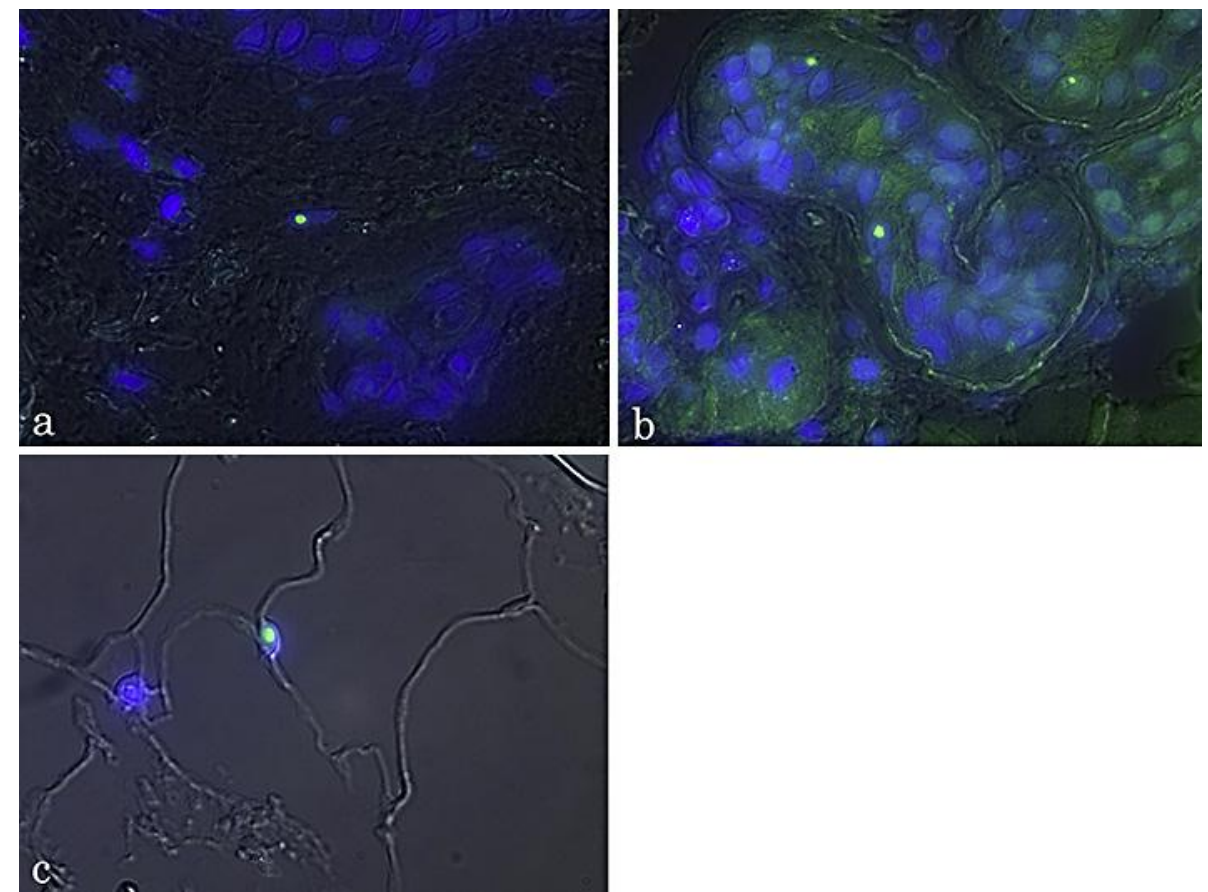

(C) 2014 S. Karger AG, Basel

www.karger.com/crn

Kitagawa et al.: Neuronal Intranuclear Inclusion Disease Presenting with Resting Tremor

Fig. 3. Double immunofluorescence staining with anti-ubiquitin antibody and 4',6-diamidino-2phenylindole dilactate (DAPI) in NIID skin samples. Intranuclear inclusions were stained with antiubiquitin antibody (green), and these inclusions were included in the DAPI-positive nuclei in the merged view. a Fibroblast. b Sweat gland cells. c Adipocytes. 